\title{
Rentabilidad económica y análisis de sensibilidad del proceso de producción de cerveza a partir de sorgo rojo CIAP R-132 a escala piloto
}

\section{Economic Profitability and Sensitivity Analysis of Beer Production Process from Red Sorghum CIAP R-132 at Pilot Scale}

\section{Amaury Pérez Sánchez ${ }^{\mathrm{ab}}$, Delcio Venâncio de Magalhães Malengue ${ }^{\mathrm{ac}}$, Isnel Benitez Cortés ${ }^{\text {ad }}$}

\footnotetext{
a Departamento de Ingeniería Química, Facultad de Ciencias Aplicadas, Universidad de Camagüey “Ignacio Agramonte Loynaz", Cuba

b amaury.psanchez@reduc.edu.cu https://orcid.org/0000-0002-0819-6760

c https://orcid.org/0000-0002-1534-0927

${ }^{d}$ https://orcid.org/0000-0003-2427-640X
}

\section{RESUMEN}

En el presente trabajo se efectuó la simulación de una planta de producción de cerveza a escala piloto (150 L/lote), empleando sorgo rojo CIAP R-132 como materia prima principal. Se efectuó un estudio de sensibilidad consistente en 11 corridas experimentales, mediante el cual se evaluó la influencia de tres variables iniciales (capacidad de producción de cerveza por lote, costo de adquisición del sorgo rojo y precio de venta de la botella de cerveza) sobre tres indicadores económicos de importancia: valor actual neto (VAN), tasa interna de retorno (TIR) y período de recuperación de la inversión (PRI). Se deben invertir $\$ 570.000$ pesos cubanos (CUP) para erigir la planta de producción, el costo unitario de producción alcanzó un valor de CUP 12,82/ botella, mientras que se obtuvo un valor de VAN, TIR y PRI de CUP $480.000,28,83 \%$ y 3,82 años, respectivamente, lo cual califica al proyecto como económicamente rentable y factible desde el punto de vista inver-

Citation: Pérez-Sánchez, A., de Magalhães-Malengue, D. C. y BenitezCortés, I. (2020). Rentabilidad económica y análisis de sensibilidad del proceso de producción de cerveza a partir de sorgo rojo ciAp R-132 a escala piloto. Mutis, 10(2), 10-24. https://doi.org/10.21789/22561498.1665

Recibido: Febrero 7, 2020. Aceptado: Junio15, 2020.

Copyright: @2020. Pérez-Sánchez, A., de Magalhães-Malengue, D. C. y Benitez-Cortés, I. This is an open-access article, which permits unrestricted use, distributions and reproduction in any medium, provided the original author and source are credited.

Competing Interests: The authors have no conflict of interest. sionista. Se obtuvieron ecuaciones que establecen la correlación estadística existente entre las tres variables de entrada y las tres de salida. Se empleó el simulador SuperPro Designer ${ }^{\circledR}$ v.8.5 para efectuar la simulación y el software Statgraphics Centurion $\mathrm{XVI}^{\circledR}$ para el procesamiento estadístico de los datos.

Palabras clave: análisis de sensibilidad, cerveza, rentabilidad económica, simulación, sorgo. 


\section{ABSTRACT}

This work presents the simulation of a pilot scale beer production plant ( $150 \mathrm{~L} / \mathrm{batch})$ with red sorghum CIAP R-132 as the main raw material. A sensitivity study consisting of 11 experimental runs was carried out, through which the influence of three initial variables (beer production capacity per batch, purchasing cost of red sorghum, and sale price of the beer bottle) was evaluated over three important economic indicators: Net present value (NPV), internal rate of return (IRR), and payback time (PT). Results show that $\$ 570,000$ Cuban pesos (CUP) must be invested to erect the production plant, the unit cost of production reached a value of cup 12.82/bottle, while NPV, IRR and PT values were CUP $480,000,28.83 \%$ and 3.82 years, respectively, which qualifies the project as economically profitable and feasible from the investor's point of view. Several equations were obtained that establish the statistical correlation between the three input variables and the three output variables. The SuperPro Designer ${ }^{\circledR}$ v.8.5 simulator was used to carry out the simulation, as well as Statgraphics Centurion XVI $^{\circledR}$ software for the statistical processing of data.

Keywords: Sensitivity analysis, beer, economic profitability, simulation, sorghum.

\section{INTRODUCCIÓN}

En la actualidad, la industria química cubana debe basar su desarrollo en investigaciones hacia la búsqueda de productos que puedan resultar atractivos desde el punto de vista de su uso, calidad y mercado, lo cual conllevaría también a su factibilidad técnica, económica y ambiental (Nieblas et al., 2016).

El sorgo constituye el quinto cereal más importante en el mundo, después del arroz, el trigo, el maíz y la avena, siendo el principal cereal alimenticio para alrededor de 750 millones de personas viviendo en regiones tropicales semiáridas de África, Asia y Latinoamérica (Ramatoulaye et al., 2016). Este cereal contiene varias propiedades beneficiosas y se ha demostrado que su cultivo es económicamente rentable, teniendo en cuenta su bajo costo de producción, resistencia a la sequía y altas temperaturas, dadas sus características de rusticidad, y la realización de varias cosechas o cortes, además de no contener el gluten que afecta a los enfermos celíacos, por lo que se constituye en una excelente alternativa para la sustitución de importaciones (Rodríguez et al., 2015).

La cerveza es una expresión genérica para designar a la bebida resultante de fermentar, mediante levadura seleccionada, el mosto procedente de la malta de granos de cereal (solo o mezclado con otros adjuntos cerveceros transformables en azúcares por digestión enzimática). Esta bebida es sometida previamente a un proceso de cocción y aromatización de sus extractos o concentrados con flores de lúpulo (Gallardo et al., 2013).

En la Universidad Central "Marta Abreu" de Las Villas, Cuba, se ha trabajado por varios años en el desarrollo y la obtención de productos a partir del sorgo, entre los que se encuentran la producción de jarabes dextrinizados (Rodríguez et al., 2015), maltina (Díaz, 2014; Gallardo et al., 2013), cerveza (Carvajal, 2014; Nieblas et al., 2016; Ortega, 2016; Pino, 2017) y etanol (Gallardo et al., 2011).

La simulación de procesos para la evaluación de alternativas y la mitigación de pérdidas en un proceso determinado juega un papel muy importante para obtener el modelo final de la simulación, ya que este puede revelar a gran escala si un proceso futuro puede dar resultados acertantes (o no) en cuanto a su factibilidad técnica y económica (Auli et al., 2013). La simulación de una planta química consiste en la creación de un modelo de proceso o de costo, entendiéndose por modelo una descripción del comportamiento de un proceso real, el cual sea capaz de predecir la salida (esto es, las respuestas) en función de las entradas. Entre los múltiples simuladores de procesos existentes en la actualidad, SuperPro Designer ${ }^{\circ}$ es uno de los más relevantes para la industria de procesos químicos, siendo empleado fundamentalmente para la 
realización de balances de masa, estudios de factibilidad y análisis económicos de variantes, así como en el diseño y la evaluación conceptual de procesos y plantas químicas (Aguiar et al., 2018; Csighy et al., 2017; Govindarajan et al., 2019; Lam et al., 2014; Mani et al., 2016).

Entre los indicadores más importantes para evaluar un proyecto en ingeniería química desde el punto de vista económico se encuentran el valor actual neto (VAN), la tasa interna de retorno (TIR) y el período de recuperación de la inversión (PRI) (Sayar et al., 2019). EI VAN da cuenta del valor del dinero en el tiempo y es la diferencia entre el valor presente de todos los flujos de caja positivos (presentes y futuros) y los flujos de salida al contado, tales como la inversión de capital; se trata de una medida de la factibilidad del proceso propuesto. La TIR no es más que la tasa de descuento que hace igual a cero el valor del van a lo largo de un tiempo predeterminado de vida del proyecto. Este constituye generalmente una base comparativa excelente para evaluar el proyecto en el ambiente económico local. Por último, el PRI se refiere a la duración requerida para que los ingresos totales igualen la inversión de capital inicial (Peters et al., 2003).

El análisis de sensibilidad se emplea para determinar la manera en que determinado proceso reacciona a cambios en variables claves, tanto de diseño como de operación, del proceso bajo estudio. Este análisis presenta múltiples beneficios, entre los que sobresalen el estudio de la influencia de los cambios efectuados en variables de entrada sobre variables de salida, verificar que es factible la solución a las especificaciones de diseño, representar gráficamente las influencia de las variables iniciales y estudiar las variables que fluctúan en el tiempo a través de un método en estado cuasi-estacionario (Ibrahim et al., 2020). Hasta la fecha, son varios los autores que han efectuado estudios de sensibilidad en diversos procesos químicos, entre los cuales se pueden mencionar el diseño de un absorbedor para la captura de dióxido de carbono utilizando aminas (Razi et al., 2013), la evaluación técnica y económica del proceso de producción de biodiésel (Tang et al., 2015), la producción de Heparinasa I expresada en Escherichia coli (Ernst et al., 1997) y la producción de etanol a partir de biomasa lignocelulósica (Rao, 2005).
El sorgo rojo, variedad CIAP R-132, ha sido utilizado para evaluar el proceso de molienda en húmedo de la extracción del almidón contenido en los granos (Rodríguez et al., 2015); la producción de jarabes dextrinizados mediante hidrólisis enzimática del almidón, aplicando la enzima $\alpha$-amilasa (Rodríguez et al., 2015); como componente adjunto mezclado con cebada para la obtención de cerveza y malta a escala piloto (50 L) (Nieblas et al., 2016); y como única materia prima para la obtención de cerveza a escala de laboratorio (Alfonso, 2018). Sin embargo, hasta la fecha, no se ha reportado su empleo de forma integral para la producción de cerveza a escala de planta piloto, por lo que no se conoce qué valores tendrán los principales indicadores técnicos y económicos de una planta de este tipo.

Considerando los elementos hasta aquí descritos, el objetivo general del presente trabajo consiste en determinar la rentabilidad técnica y económica del proceso de producción de cerveza a partir de sorgo rojo CIAP R-132 a escala de planta piloto (150 L/lote) en el contexto económico actual de Cuba. Además, se efectúa un estudio de sensibilidad con el fin de evaluar la influencia que presentan tres variables iniciales, (i) capacidad de producción de cerveza por lote, (ii) costo de adquisición del sorgo rojo y (iii) precio de venta de la botella de cerveza, sobre tres importantes parámetros económicos del proceso de producción evaluado: VAN, TIR y PRI. Por último, se determinó la correlación estadística existente entre las tres variables iniciales seleccionadas y los parámetros económicos VAN, TIR y PRI, para así obtener correlaciones que describan cuantitativamente la relación estadística existente entre estas variables.

\section{MATERIALES Y MÉTODOS}

\section{Descripción del proceso de producción de cerveza a partir de sorgo rojo CIAP R-132}

En la figura 1 se muestra el diagrama de bloques en los que se exponen las principales etapas del proceso de producción de cerveza a partir de sorgo rojo CIAP R-132. 


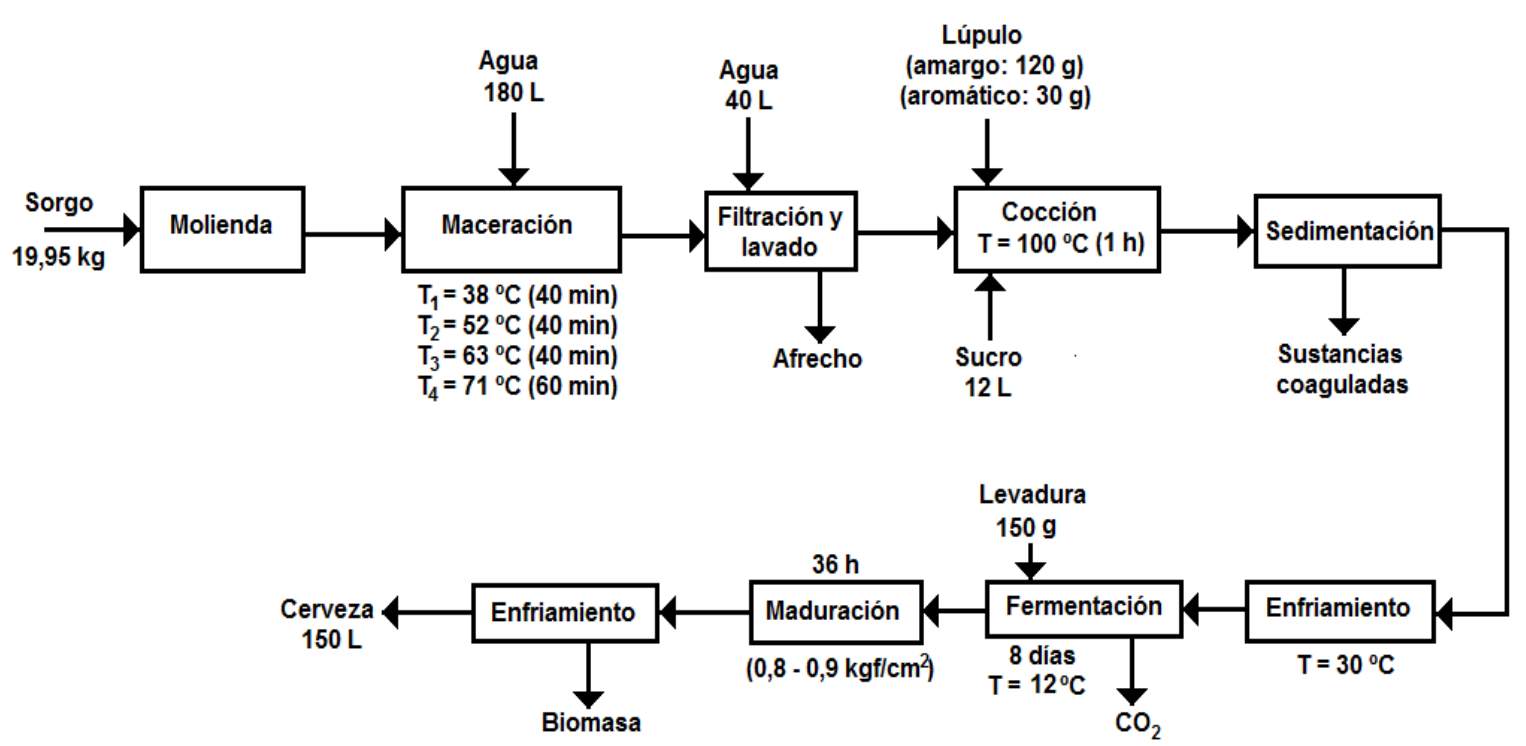

Figura 1. Diagrama de bloques del proceso de producción de cerveza a partir de sorgo rojo CIAP r-132.

Fuente: elaboración propia.

Este proceso cuenta con dos subetapas principales, siendo la primera la subetapa en caliente donde se realizan las siguientes operaciones:

1. Molienda

2. Maceración

3. Filtración y lavado

4. Cocción del mosto

5. Sedimentación

La segunda subetapa, conocida como etapa en frío, consta de las siguientes etapas:

1. Enfriamiento

2. Inyección de la levadura y fermentación

3. Maduración

4. Enfriamiento, reposo de la cerveza y extracción de la levadura

5. Envasado

En este caso, el proceso en caliente tiene como objetivo principal disolver las sustancias de la malta y los sucedáneos empleados, desdoblándolos en azúcares fermentables para obtener un mosto libre de contaminación y de sustancias insolubles que sea adecuado para ser fermentado. En esta etapa del proceso ocurren una serie de operaciones con los objetivos descritos a continuación.

\section{Molienda}

Se trituran 19,95 kg de sorgo en un molino de disco para reducir su tamaño hasta obtener una consistencia de harina granulada, con vistas a favorecer la posterior sacarificación del almidón durante el proceso de maceración.

\section{Maceración}

La maceración se realiza para lograr la conversión de los almidones contenidos en el sorgo en azúcares fermentables (sacarificación). Para ello, se comienza agregando $180 \mathrm{~L}$ de agua al macerador y luego los 19,95 kg de sorgo 
previamente molidos. Durante el proceso de maceración se llevan a cabo 4 pausas o escalones de temperaturas. En primer lugar, se calienta el agua contenida dentro del macerador hasta una temperatura de $38 \stackrel{\circ}{\circ}$, a partir de la cual se le agregó el grano de sorgo; esta pausa de temperatura se mantiene durante 40 minutos. Posteriormente, se incrementa la temperatura de la mezcla hasta $52 \stackrel{\circ}{\circ}$ y se mantiene en este valor también durante 40 minutos. Transcurrido este tiempo, se incrementa la temperatura del mosto hasta $63 \stackrel{\circ}{\circ}$ y se conserva durante 40 minutos. Por último, se realiza un incremento de la temperatura hasta $71 \stackrel{\circ}{ } \mathrm{C}$, permaneciendo en este valor durante 60 minutos. Cumplido este tiempo se incrementa la temperatura del líquido macerado hasta $78 \stackrel{\circ}{ } \mathrm{C}$ con el fin de detener la actividad enzimática. Todo el proceso de maceración se efectúa a una velocidad de agitación de $60 \mathrm{rpm}$. Una vez finalizado el proceso de maceración, al mosto se le aplica la prueba del yodo a fin de comprobar si existió sacarificación del almidón durante el proceso de maceración; es decir, si el almidón se desdobló en azúcares simples. En el mosto filtrado y enfriado también se mide el valor de Brix y el pH.

La reacción de hidrolisis enzimática del almidón se describe mediante las ecuaciones 1, 2 y 3 (Suárez et al., 2019):

$$
\begin{aligned}
& \left(C_{6} H_{10} O_{5}\right)_{n}+H_{2} \mathrm{O} \rightarrow n C_{6} H_{12} O_{6} \\
& 2\left(C_{6} H_{10} O_{5}\right)_{n}+H_{2} O \rightarrow n C_{12} H_{22} O_{11} \\
& C_{12} H_{22} O_{11}+H_{2} \mathrm{O} \rightarrow 2 C_{6} H_{12} O_{6}
\end{aligned}
$$

\section{Filtración y lavado}

Se procede a separar el líquido (mosto) del sólido (afrecho). Esta separación se realiza en un colador metálico a través del cual fluye el mosto filtrado, siendo almacenado en un recipiente colector metálico. A la torta de afrecho acumulada en el colador se le realiza un lavado con agua a 75-78 으 con el fin de que continúen disolviéndose los azúcares contenidos en esta mezcla sólida. El líquido de lavado es recogido en el mismo recipiente donde se colectó el mosto durante el primer filtrado. El afrecho agotado es comercializado, mientras que el mosto contenido en el recipiente colector es bombeado de vuelta al macerador.

\section{Preparación del sucro}

En un recipiente aparte se agregan 10,5 kg de azúcar y $12 \mathrm{~L}$ de agua, los cuales son esterilizados a 100 ㅇ C por 25 minutos. Una vez concluida la esterilización, la mezcla resultante (sucro) es enfriada.

\section{Cocción}

Por medio de la cocción se produce la esterilización y concentración del mosto para que este sea suministrado libre de contaminación microbiana a la etapa de fermentación. En esta operación se logra la coagulación de las sustancias insolubles y la inactivación de las enzimas que hasta este momento siguen realizando su función. En esta etapa se adicionan $120 \mathrm{~g}$ de lúpulo amargo, $30 \mathrm{~g}$ de lúpulo aromático y los $12 \mathrm{~L}$ de sucro. La cocción se realiza durante 1 hora a una temperatura de $100 \stackrel{\circ}{ }$, tras lo cual se añade agua de proceso, previamente esterilizada, hasta completar 150 litros.

\section{Sedimentación}

Una vez finalizada la cocción del mosto, este es vertido en un tanque colector metálico y bombeado hacia un sedimentador tangencial (whirlpool) para separar la mayor cantidad de partículas sólidas en suspensión que contiene el mosto caliente, la cual está formada por compuestos tanino-proteicos, globulinas y otras partículas finas provenientes del sorgo, así como también del sucro. En este caso, debido a la fuerte rotación que se produce dentro del sedimentador se reúnen o agrupan los coágulos, los cuales se sedimentan y forman un cono de sustancias coaguladas en el fondo del sedimentador, separándose de esta forma las partículas coagulantes que están en suspensión del líquido clarificado. La mezcla sedimentada es enviada para consumo animal.

Por su parte, la segunda subetapa, es decir, el proceso en frío, tiene como objetivo crear un medio adecuado para la fermentación, lograr una fermentación acelerada y obtener un producto que cumpla con las normas de calidad establecidas. Las etapas incluidas en este proceso son:

\section{Enfriamiento}

Se enfría el mosto contenido en el sedimentador hasta una temperatura de $30 \stackrel{\circ}{ } \mathrm{C}$, haciendo circular agua de enfriamiento por la chaqueta del equipo, la cual proviene de la torre de enfriamiento. 


\section{Inyección de la levadura y fermentación}

Su objetivo es lograr una fermentación acelerada y una relación adecuada del microorganismo y la cantidad de mosto a fermentar, ocurriendo la transformación de los azúcares en el mosto en alcohol etílico y dióxido de carbono por acción de la levadura. Una vez que el mosto alcanza la temperatura de 30 ㄷ en el sedimentador, este es bombeado hacia el reactor $y$, seguidamente, se inyecta la levadura. En el reactor, este compuesto es aireado con un botellón de oxígeno por espacio de una hora, aproximadamente. Toda esta operación se efectúa manteniendo la recirculación del medio fermentativo, lo que facilita la reacción en medio agitado, regulando la entrada del oxígeno necesario para el crecimiento y la propagación de la levadura.

La ecuación de fermentación alcohólica se muestra a continuación (ec. 4):

$$
\mathrm{C}_{6} \mathrm{H}_{12} \mathrm{O}_{6} \rightarrow 2 \mathrm{C}_{2} \mathrm{H}_{5} \mathrm{OH}+2 \mathrm{CO}_{2}
$$

Una vez aireada, se procede a efectuar la fermentación anaerobia para producir la cerveza, proceso que se lleva a cabo a una temperatura de $12{ }^{\circ} \mathrm{C}$. Diariamente se lleva a cabo un análisis de extracto y $\mathrm{pH}$. Cuando el extracto llega a $5^{\circ} \mathrm{Brix}$ se pasa a la etapa de maduración.

\section{Maduración}

En esta etapa la presión del fermentador aumenta hasta $0,8-0,9 \mathrm{kgf} / \mathrm{cm}^{2}$ y la temperatura se mantiene entre 18-19 o $\mathrm{C}$ para saturar la cerveza de $\mathrm{CO}_{2}$. El extracto debe ir disminuyendo paulatinamente al ser fermentado por la levadura. Así mismo, el pH debe disminuir hasta 3,5-3,8. Cuando el extracto se mantiene constante a un valor límite de fermentación se pasa a la etapa de enfriamiento en el fermentador.

\section{Enfriamiento, extracción de la levadura y reposo de la cerveza}

Durante esta etapa se enfría la cerveza hasta 0-2 ㄷ para que precipite la levadura al fondo del fermentador. Esta operación tiene como objetivo separar el resto de la cosecha de levadura sedimentada en el fondo del reactor y eliminar proteínas coagulables, partículas insolubles y sustancias coloidales que no se hayan precipitado en el proceso de fermentación, favoreciendo así la formación de compuestos que apor- tan el buqué característico de cada tipo de cerveza. En esta etapa, la mezcla se deja reposar por 24 horas y se comienza la extracción de la levadura. Posterior a la extracción se deja reposar por 12 horas. Durante esta operación se debe mantener la presión entre 0,8 y 0,9 $\mathrm{kgf} / \mathrm{cm}^{2}$ para saturar de $\mathrm{CO}_{2}$ la cerveza.

\section{Envasado}

Una vez extraída la levadura (biomasa) y efectuada la maduración de la cerveza, esta es envasada en botellas de cristal de $350 \mathrm{~mL}$ de capacidad.

\section{Simulación del proceso de producción en el sim- ulador SuperPro Designer}

Se empleó el simulador profesional de procesos SuperPro Designer ${ }^{\circ}$ v. 8.5 para simular el proceso de producción de cerveza a partir de sorgo rojo CIAP R-132, con el fin de aplicar las herramientas de balance de masa y energía, el diseño de equipamiento y los cálculos económicos del proceso bajo estudio (figura 2).

La simulación del proceso productivo en el simulador SuperPro Designer permitió obtener valores y resultados de un gran número de parámetros técnicos y económicos de importancia para este proceso de producción, entre los cuales se puede mencionar VAN, TIR, PRI, costos fijos, capital de trabajo, costo unitario de producción, margen bruto y neto y porcentaje de retorno de la inversión.

Para efectuar la simulación se asumió un tiempo de construcción de planta de 15 meses, con 3 meses para realizar la arrancada y puesta en marcha. Se consideró un tiempo de vida del proyecto de 15 años, asumiendo que la planta producirá al $100 \%$ durante todo su tiempo de vida. El VAN fue determinado asumiendo una tasa de interés de $11 \%$ y un $32 \%$ de impuestos por concepto de ingresos. El costo de validación y puesta en marcha se asumió como $15 \%$ de los costos fijos directos (CFD), mientras que los costos asociados con el aseguramiento y control de la calidad se suponen como un $15 \%$ del costo total de la mano de obra. Se asume además que no existe rechazo del producto principal (cerveza) por no cumplir con los parámetros de calidad exigidos, que se gastan alrededor de CuP 20.000 anuales para efectuar operaciones de validación del proceso de producción y que el costo relacionado con el tratamiento de residuales corresponde a $25 \%$ del costo total de operación. 


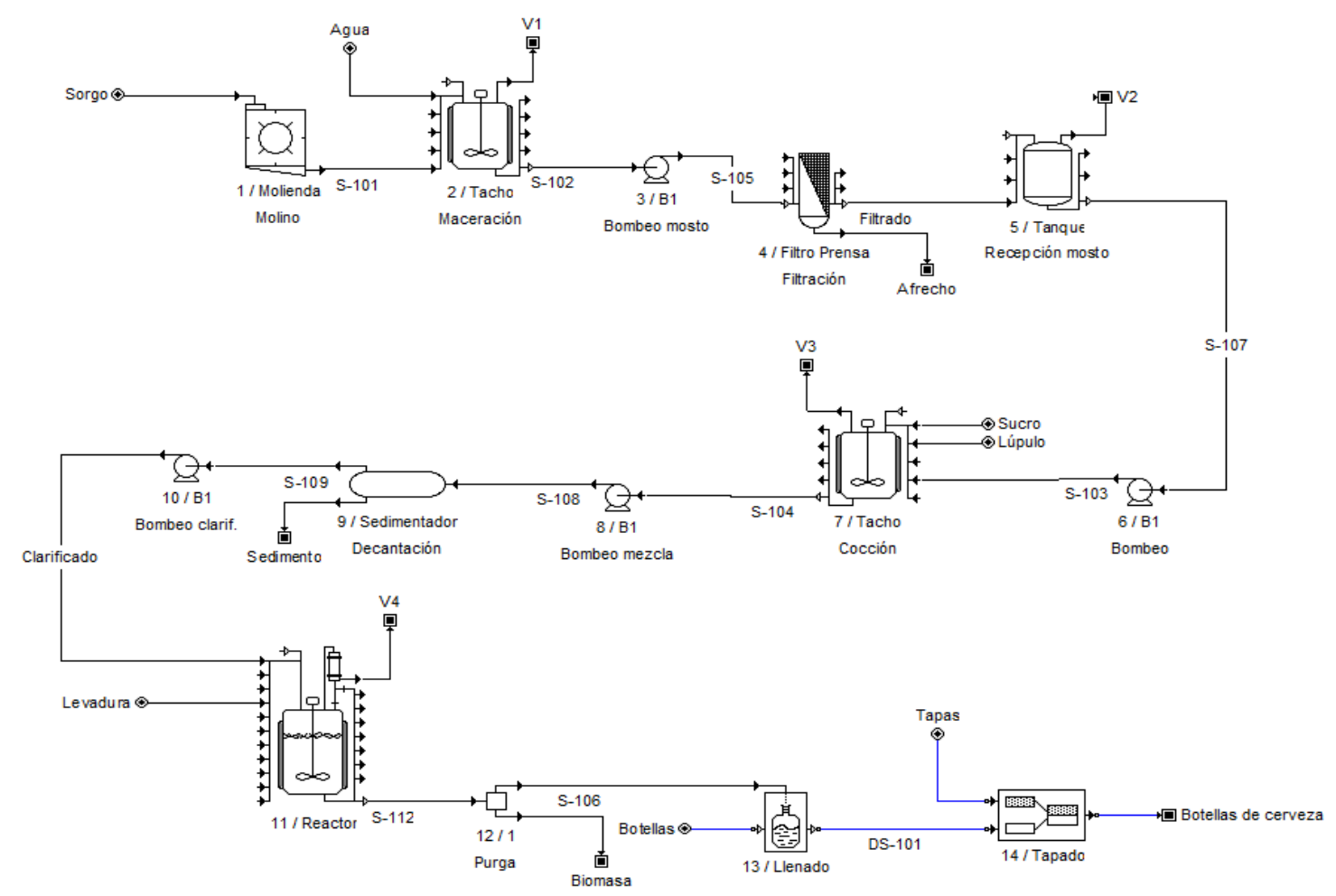

Figura 2. Diagrama de flujo del proceso de producción de cerveza a partir de sorgo rojo CIAP R-132 obtenido mediante el simulador SuperPro Designer ${ }^{\circledast}$.

Fuente: elaboración propia.

La planta utiliza todos los servicios auxiliares comúnmente consumidos en un proceso de este tipo, es decir, agua de enfriamiento, glicol, electricidad y vapor de agua. Se asume además que la misma trabaja 11 meses al año, con 1 mes para acometer operaciones de mantenimiento y reparaciones de equipos y sistemas auxiliares. Se aplicó un salario nominal promedio de CUP 2,84/hora para los operarios que trabajan en la planta y CUP 5,00/hora para los supervisores y personal de dirección. La tabla 1 muestra los precios de las principales materias primas consumidas, los materiales utilizados, los servicios auxiliares empleados y los productos obtenidos durante el proceso productivo.

En la tabla 2 se muestran los costos de adquisición de cada equipo empleado en el proceso productivo, los cuales fueron tomados de varias fuentes y referencias bibliográficas (Perry \& Green, 2008; Peters et al., 2003; Towler \& Sinnott, 2013; Sinnott \& Towler, 2019). El producto final obtenido (cerveza) se envasará en botellas de cristal de $350 \mathrm{~mL}$ de capacidad.
Tabla 1. Precios de las principales materias primas, los servicios auxiliares y los productos obtenidos utilizados en la simulación del proceso productivo (cifras en pesos cubanos [CUP])

\begin{tabular}{|c|c|c|}
\hline Compuesto & Precio & Unidad \\
\hline Sorgo rojo CIAP R-132 & 14,05 & CUP/kg \\
\hline Agua & 0,10 & CUP/m ${ }^{3}$ \\
\hline Lúpulo amargo & 51,228 & CUP/kg \\
\hline Lúpulo aromático & 65,31 & CUP $/ \mathrm{kg}$ \\
\hline Azúcar crudo & 356,15 & CUP/t \\
\hline Afrecho & 60 & CUP/t \\
\hline Levadura & 50 & CUP/t \\
\hline Agua de enfriamiento & 0,05 & CUP/t \\
\hline Glicol & 0,40 & CUP/t \\
\hline Vapor de agua & 3,50 & CUP/t \\
\hline Electricidad & 1,20 & CUP/kWh \\
\hline Botella $350 \mathrm{~mL}$ & 0,306 & CUP/Ud \\
\hline Tapas & 0,007 & CUP/Ud \\
\hline Cerveza envasada en botellas & 24 & CUP/botella \\
\hline de $350 \mathrm{~mL}$ & &
\end{tabular}

Fuente: elaboración propia. 
Tabla 2. Costo del equipamiento principal empleado en el proceso de producción de cerveza a partir de sorgo rojo CIAP R-132

\begin{tabular}{|c|c|c|c|}
\hline Equipo & Características & Cantidad & $\begin{array}{c}\text { Costo total } \\
\text { (CUP) }\end{array}$ \\
\hline Molino de discos & $40 \mathrm{~kg} / \mathrm{h}$ & 1 & 10.200 \\
\hline Macerador & $200 \mathrm{~L}$ & 1 & 13.000 \\
\hline Filtro colador & $25 \mathrm{~m}^{2}$ & 1 & 3.000 \\
\hline Tanque recepción mosto & $200 \mathrm{~L}$ & 1 & 10.000 \\
\hline Sedimentador & $200 \mathrm{~L}$ & 1 & 14.300 \\
\hline Reactor & $200 \mathrm{~L}$ & 1 & 15.700 \\
\hline Bomba centrífuga sanitaria & $3,5 \mathrm{~kW}$ & 1 & 4.900 \\
\hline \multicolumn{2}{|c|}{ Total } & & $\mathbf{7 1 . 1 0 0}$ \\
\hline
\end{tabular}

Fuente: elaboración propia.

La tabla 3 muestra la composición química porcentual del sorgo y el lúpulo utilizados durante la simulación, datos obtenidos de Díaz (2014) y Pérez (2016), respectivamente. Por último, se escoge una capacidad promedio de producción de la planta de 150 litros de cerveza por lote.

Tabla 3. Composición química porcentual del sorgo y el lúpulo utilizados en la simulación

\begin{tabular}{|c|c|c|}
\hline Componente & Sorgo (\%) & Lúpulo (\%) \\
\hline Humedad & 10,6 & 10,0 \\
\hline Almidón & 69,3 & - \\
\hline Proteína & 12,5 & 15,0 \\
\hline Grasa & 3,4 & - \\
\hline Fibra & 2,2 & - \\
\hline Cenizas & 2,0 & 3,0 \\
\hline Resinas & - & 15,0 \\
\hline Taninos & - & 4,0 \\
\hline Alfa-ácidos & - & 8,0 \\
\hline Lignina & - & 45,0 \\
\hline
\end{tabular}

Fuente: elaboración propia con base en Díaz (2014) y Pérez (2016).

\section{Evaluación de la sensibilidad de los indicadores seleccionados}

Considerando los resultados técnico-económicos obtenidos durante la simulación del proceso productivo a través del simulador SuperPro Designer ${ }^{\circ}$, se desarrolló un diseño de experimentos estadísticos del tipo
Superficie de Respuesta por medio del software estadístico Statgraphics Centurion ${ }^{\circ}$ XVI para evaluar la influencia de tres variables iniciales o de entrada (capacidad de producción de cerveza por lote, costo de adquisición del sorgo rojo y precio de venta de la botella de cerveza) sobre tres importantes parámetros de salida de proceso: VAN, TIR y PRI. De esta manera, se llevará a cabo un estudio de sensibilidad de 3 entradas y 3 salidas.

Para elaborar el diseño de experimentos descrito, a los valores utilizados durante la simulación para las 3 variables iniciales consideradas se le aumentará o disminuirá $20 \%$. Lo anterior con el objetivo de tomar en cuenta posibles variaciones u oscilaciones de los valores que puedan presentar estas variables en el futuro y evaluar la potencial influencia tales fluctuaciones en los resultados de VAN, TIR y PRI para la planta propuesta.

Se elaboró un diseño de experimentos del tipo superficie de respuesta aleatorizado aplicando la opción "Box-Behnken Design", contenida en el paquete estadístico Statgraphics Centurion", obteniendo inicialmente 30 corridas experimentales en total. Estas últimas fueron posteriormente optimizadas mediante la herramienta "D-Optimality", contenida en el propio software estadístico, con el objetivo de seleccionar aquellas corridas que presentan mayor influencia estadística sobre las 3 variables de salida tomadas en cuenta, así como también reducir la extensión del estudio de sensibilidad, llegando finalmente a 11 corridas experimentales. En la tabla 4 se muestran los valores que deben presentar cada una de las 3 variables de entrada tomando en cuenta el rango de $\pm 20 \%$. Por su parte, la tabla 5 expone los valores que deberán presentar estos 3 parámetros dentro del diseño de experimentos optimizado que contiene las 11 corridas experimentales.

Tabla 4. Valores que deben presentar cada una de las 3 variables de entrada consideradas tomando en cuenta un rango de variación de $\pm 20 \%$

\begin{tabular}{|c|c|c|c|}
\hline Parámetro & $\begin{array}{c}\text { Valor } \\
\text { utilizado }\end{array}$ & $\begin{array}{c}\text { Valor } \\
\text { mínimo } \\
(-\mathbf{2 0} \%)\end{array}$ & $\begin{array}{c}\text { Valor } \\
\text { máximo } \\
(+\mathbf{2 0} \%)\end{array}$ \\
\hline Capacidad de producción (L/lote) & 150 & 120 & 180 \\
\hline Costo de compra sorgo (cup/kg) & 14,05 & 11,24 & 16,86 \\
\hline Precio de venta cerveza (cUP/kg) & 24 & 19,2 & 28,8 \\
\hline
\end{tabular}

Fuente: elaboración propia. 
Tabla 5. Estudio de sensibilidad optimizado que contiene los valores a considerar de cada parámetro de entrada

\begin{tabular}{|c|c|c|c|}
\hline Corrida & $\begin{array}{c}\text { Capacidad de } \\
\text { producción (L/lote) }\end{array}$ & $\begin{array}{c}\text { Costo de compra } \\
\text { sorgo (cup/kg) }\end{array}$ & $\begin{array}{c}\text { Precio de venta cerveza } \\
\text { (cup/botella) }\end{array}$ \\
\hline 1 & 180 & 11,24 & 28,8 \\
\hline 2 & 180 & 11,24 & 19,2 \\
\hline 3 & 180 & 16,86 & 19,2 \\
\hline 4 & 120 & 16,86 & 19,2 \\
\hline 5 & 120 & 16,86 & 28,8 \\
\hline 6 & 180 & 11,24 & 19,2 \\
\hline 7 & 120 & 16,86 & 19,2 \\
\hline 8 & 120 & 11,24 & 28,8 \\
\hline 9 & 180 & 16,86 & 28,8 \\
\hline 10 & 120 & 16,86 & 19,2 \\
\hline 11 & 120 & 11,24 & 19,2 \\
\hline
\end{tabular}

Fuente: elaboración propia.

Evaluación de la correlación estadística existente entre las variables de entrada y los indicadores VAN, TIR Y PRI

Una vez efectuado el estudio de sensibilidad, se procedió a realizar un análisis de la correlación estadística existente entre las tres variables de entrada consideradas y los tres parámetros económicos de salida evaluados, con el objetivo de obtener correlaciones o ecuaciones que describan, de forma cuantitativa, la relación estadística existente entre las variables de entrada y las de salida. Lo anterior se llevó a cabo utilizando la opción "Multiple Regression" contenida en el software estadístico Statgraphics Centurion ${ }^{\circ} \mathrm{XVI}$.

Por último, se determinó la corrida (o escenario) con el resultado económico más positivo con respecto a los resultados de VAN, TIR y PRI obtenidos, así como también aquella con el resultado menos favorable.

\section{RESULTADOS Y DISCUSIÓN}

\section{Principales resultados técnico-económicos ob- tenidos durante la simulación del proceso de producción de cerveza a escala piloto}

La tabla 6 presenta los principales indicadores técnicos y económicos obtenidos durante la simulación del proceso de producción de cerveza en el simulador SuperPro Designer ${ }^{\circ}$.
Tomando en cuenta los resultados mostrados en la tabla 6, para una planta con una inversión inicial de cuP 570000 , el proyecto se puede calificar como económicamente rentable y confiable desde el punto de vista inversionista, puesto que el PRI no es superior a 5 años (3,82 años), la TIR es mayor a $25 \%(28,83 \%)$ y el VAN presenta un resultado positivo (CUP 480000 ), lo cual es un indicativo de rentabilidad de la inversión y ganancias positivas (Baca, 2010; Sinnott \& Towler, 2019; Towler \& Sinnott, 2013).

Estudios previos relacionados con la evaluación económica de plantas de producción de cerveza empleando sorgo como materia prima obtuvieron los resultados que se muestran a continuación. Carvajal (2014) dimensionó una planta piloto de producción de cerveza empleando sorgo blanco UDG-110 como materia prima, con una capacidad de producción de 100 L/día, obteniendo un costo total de la inversión de CUP $106.965,18$, un costo total de producción de CUP $16.755,44$ /año, una ganancia anual de CUP 28.436,56, un VAN de CUP $75.410,73$, una TIR de $24 \%$ y un PRI de 5,5 años. Por su parte, Ortega (2016) diseñó una planta piloto de producción de cerveza de 100 L/día con sorgo blanco UDG-110 como materia prima principal, obteniendo como resultado un VAN de CUP 1.905.901,7, una TIR de $40 \%$ y un PRI de cuatro años y seis meses. Por último, Pino (2017) efectuó el diseño de una planta de producción de cerveza con la misma variedad de sorgo y capacidad de producción, obteniendo los siguientes resultados: VAN de CUP $14.760 .706,1$, TIR de $250,1 \%$ y PRI de 1 año. 
Tabla 6. Principales indicadores técnico-económicos obtenidos durante la simulación del proceso de producción de cerveza en el simulador SuperPro Designer ${ }^{\circ}$

\begin{tabular}{|c|c|}
\hline Indicador & Valor \\
\hline Inversión total de capital (CuP) & 570.000 \\
\hline Costo de operación (CUP/año) & 187.000 \\
\hline Ganancias totales anuales (cup/año) & 351.000 \\
\hline Costo unitario de producción (cup/botella) & 12,82 \\
\hline Retorno de la inversión (CuP) & 26,15 \\
\hline Periodo de retorno de la inversión (años) & 3,82 \\
\hline Tasa interna de retorno (\%) & 28,83 \\
\hline Valor actual neto (CUP) & 480.000 \\
\hline \multicolumn{2}{|c|}{ Costo total directo de la planta (CTDP) } \\
\hline Costo de compra del equipamiento (CUP) & 89.000 \\
\hline Instalación (CUP) & 37.000 \\
\hline Tuberías (CUP) & 31.000 \\
\hline Instrumentación (CUP) & 36.000 \\
\hline Aislamiento (CUP) & 3.000 \\
\hline Sistemas eléctricos (CUP) & 9.000 \\
\hline Edificaciones (CUP) & 40.000 \\
\hline Mejora de terreno (CuP) & 13.000 \\
\hline Instalaciones auxiliares (CUP) & 36.000 \\
\hline Total CTDP (CUP) & 294.000 \\
\hline \multicolumn{2}{|c|}{ Costo total indirecto de la planta (CTIP) } \\
\hline Ingeniería (CUP) & 73.000 \\
\hline Construcción (CUP) & 102.000 \\
\hline Pagos al contratista (CUP) & 23.000 \\
\hline Contingencias (CUP) & 47.000 \\
\hline Total CTIP (CUP) & 245.000 \\
\hline Capital fijo directo (CFD) = CTDP + CTIP (CUP) & 539.000 \\
\hline \multicolumn{2}{|l|}{ Otros } \\
\hline Capital de Trabajo (CUP) & 5.000 \\
\hline Costo de arrancada (CUP) & 27.000 \\
\hline Producción anual de cerveza (botellas/año) & 14.615 \\
\hline Ganancia bruta anual (cup/año) & 42.000 \\
\hline Ganancia neta anual (cup/año) & 76.000 \\
\hline Margen en bruto (\%) & $18,51 \%$ \\
\hline Gastos anual por salario (cup/año) & 39.000 \\
\hline Gasto anual por materia prima (CuP/año) & 10.000 \\
\hline Gasto por material gastable (cuP/año) & 32.000 \\
\hline Gasto anual por consumo de servicios auxiliares (cup/año) & 1.000 \\
\hline Tiempo del lote (h) & $241,72 \mathrm{~h}$ \\
\hline Número total de lotes/año & 32 lotes/año \\
\hline
\end{tabular}

Fuente: elaboración propia. 
La tabla 7 muestra el desglose de las principales mainfluencia porcentual en el costo total de producción. terias primas y materiales consumidos por año y su

Tabla 7. Consumo de materias primas y materiales, y su influencia porcentual en el costo de producción

\begin{tabular}{|c|c|c|c|}
\hline Materia prima o material & Cantidad consumida anual & $\begin{array}{c}\text { Costo anual } \\
\text { (CUP) }\end{array}$ & $\%$ \\
\hline Sorgo & $638 \mathrm{~kg}$ & 8.970 & 63,01 \\
\hline Agua & $6 \mathrm{~m}^{3}$ & 1 & 0,00 \\
\hline Sacarosa & $336 \mathrm{~kg}$ & 120 & 0,84 \\
\hline Lúpulo & $5 \mathrm{~kg}$ & 559 & 3,93 \\
\hline Levadura & $5 \mathrm{~kg}$ & 11 & 0,08 \\
\hline Botellas & $14.615 \mathrm{Ud}$ & 4.472 & 31,42 \\
\hline Tapas & $14.615 \mathrm{Ud}$ & 102 & 0,72 \\
\hline
\end{tabular}

Fuente: elaboración propia.

\section{Evaluación del estudio de sensibilidad efectuado con respecto a las variables iniciales seleccionadas}

La tabla 8 muestra los resultados del estudio de sensibilidad efectuado para las 11 corridas consideradas.

Tabla 8. Resultados obtenidos para cada una de las corridas experimentales incluidas en el estudio de sensibilidad efectuado

\begin{tabular}{|c|c|c|c|}
\hline Corrida & $\begin{array}{c}\text { VAN } \\
\text { (CUP) }\end{array}$ & $\begin{array}{c}\text { TIR } \\
\text { (\%) }\end{array}$ & $\begin{array}{c}\text { PRI } \\
\text { (años) }\end{array}$ \\
\hline 1 & 941.283 & 33,67 & 3,15 \\
\hline 2 & 216.285 & 11,48 & 5,46 \\
\hline 3 & 197.542 & 11,17 & 5,57 \\
\hline 4 & -44.451 & 5,70 & 7,67 \\
\hline 5 & 426.759 & 17,58 & 4,02 \\
\hline 6 & 216.285 & 11,48 & 5,46 \\
\hline 7 & -44.451 & 5,70 & 7,67 \\
\hline 8 & 439.254 & 17,89 & 3,97 \\
\hline 9 & 922.540 & 32,36 & 3,28 \\
\hline 10 & -44.451 & 5,70 & 7,67 \\
\hline 11 & -31.018 & 6,02 & 7,49 \\
\hline
\end{tabular}

Fuente: elaboración propia.

Un análisis de la información en la tabla 8 permite establecer que la corrida $n .^{\circ} 1$ presenta el escenario económico más optimista y positivo en cuanto a los mayores valores de VAN y TIR y el menor valor de PRI, con CUP 941.283 , 33,67 \% y 3,15 años, respectivamente. Esto se debe a que esta corrida presenta la mayor capacidad de producción (180 L/lote) con el mayor precio de venta de la cerveza (cup 28,8/botella), así como el menor costo de compra del sorgo rojo (CUP $11,24 / \mathrm{kg}$ ); es decir, se produce a la mayor capacidad de producción posible adquiriendo la principal materia prima consumida en el proceso (sorgo rojo) al menor precio de compra posible y vendiendo el principal producto obtenido (botella de cerveza) al mayor costo de venta posible, lo cual influye positivamente en la rentabilidad global del proceso productivo.

Por otro lado, las corridas n. 4, 7 y 10 presentaron el peor escenario económico, con un VAN de CUP -44.451, una TIR de 5,70 \% y un PRI de 7,67 años. Lo anterior se debe a que en las mismas se tiene el mayor costo de compra del sorgo (CUP $16,86 / \mathrm{kg}$ ), así como el menor precio de venta de la cerveza (cup 19,2/botella) para la menor capacidad de producción posible de la planta (120 L/lote).

Vale destacar que la corrida 11 también presentó resultados negativos con respecto a los valores de VAN (CUP -31.018), TIR $(6,02 \%)$ y PRI $(7,49)$, lo cual se debe a que esta corrida presenta la menor capacidad de producción de cerveza posible (120 L/lote) y el menor precio de venta de la cerveza (cup 19,2/botella).

\section{Resultados obtenidos con respecto a la correl- ación estadística existente entre las tres vari- ables de entrada y los indicadores VAN, TIR Y PRI}

Al evaluar la correlación estadística existente entre las tres variables de entrada consideradas (capacidad de producción de cerveza por lote, costo de adquisición del sorgo rojo y precio de venta de la botella de cerveza) sobre tres indicadores económicos importantes del proceso (VAN, TIR y PRI), se obtuvo una ecuación multifactorial para cada uno de los tres indicadores evaluados, las cuales se muestran a continuación. 
La figura 3 muestra el diagrama de Gantt obtenido mediante el empleo del simulador SuperPro Designer ${ }^{\circledR}$.

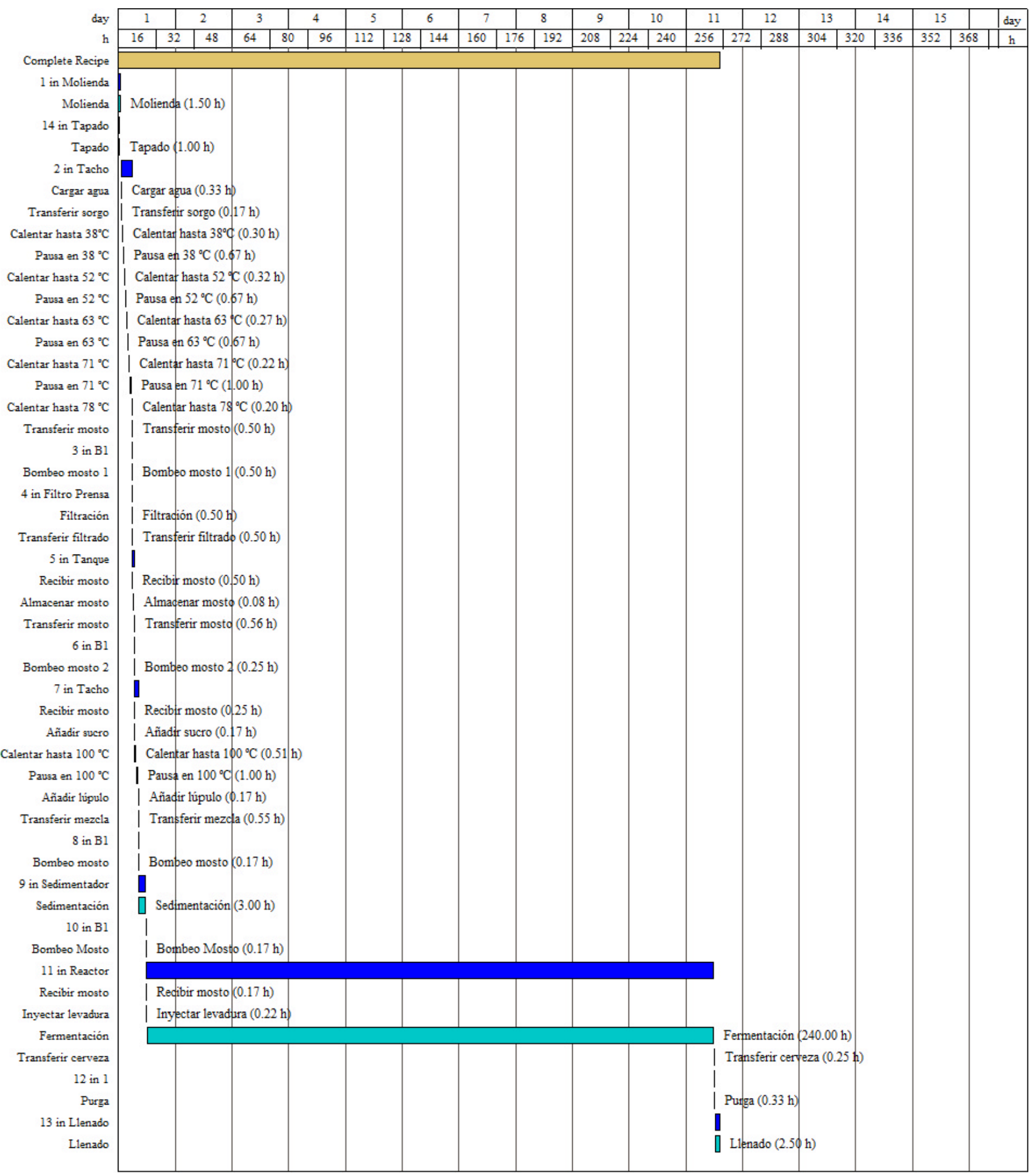

Figura 3. Diagrama de Gantt del proceso de producción de cerveza a partir de sorgo rojo CIAP R-132 Fuente: elaboración propia mediante el simulador SuperPro Designerø. 


\section{Valor actual neto (VAN)}

VAN $=-1,84541 \mathrm{E} 6+5331,67^{*} \mathrm{C}+890,517 * \mathrm{CS}+59569,7 * \mathrm{PC}$

Tasa interna de retorno (TIR) [T3]

$\operatorname{TIR}=-28,7312+0,0931736 * \mathrm{C}-0,0498962 * \mathrm{CS}+1,25282 * \mathrm{PC}$

Período de recuperación de la inversión (PRI)

$\mathrm{PRI}=15,9756-0,0264757 * \mathrm{C}+0,0415555 * \mathrm{CS}-0,312782 * \mathrm{PC}$
Donde:

C = Capacidad

CS $=$ Costo de compra de sorgo

$\mathrm{PC}=$ Precio de venta de la cerveza

La tabla 9 muestra los resultados estadísticos obtenidos para cada uno de los indicadores económicos incluidos en el estudio de correlación estadística efectuado.

Tabla 9. Resultados estadísticos obtenidos durante el estudio de correlación estadística realizado

\begin{tabular}{|c|c|c|c|}
\hline Parámetro & VAN & TIR & PRI \\
\hline R-cuadrada (\%) & 97,7517 & 99,9114 & 98,0787 \\
\hline R-cuadrado (ajustado para g.I.) & 96,7882 & 99,8734 & 94,3981 \\
\hline Error estándar & 501137 & 1,1411 & 1,3807 \\
\hline Error absoluto medio & 389393 & 0,8350 & 0,9592 \\
\hline Estadístico Durbin-Watson & $2,1785(P=0,6812)$ & $2,2796(P=0,7439)$ & $1,9050(P=0,4904)$ \\
\hline Autocorrelación de residuos en retraso 1 & $-0,1778$ & $-0,2032$ & $-0,0050$ \\
\hline
\end{tabular}

Fuente: elaboración propia.

\section{CONCLUSIONES}

El proceso de producción de cerveza a partir de sorgo rojo CIAP R-132 a escala piloto (150 L/lote) puede considerarse de rentable desde el punto de vista técnico y económico considerando los valores de VAN (CUP $480.000)$, TIR (28,83 \%) y PRI (3,82 años) obtenidos en este trabajo.

La corrida que mejor escenario económico presentó durante el estudio de sensibilidad efectuado fue la $\mathrm{n} .^{\circ}$ 1 , con una VAN = CUP 941.283, una TIR $=33,67 \%$ y un $\mathrm{PRI}=3,15$ años, mientras que las de peor resultado económico fueron las $n .^{\circ} 4,7$ y 10 , con un VAN = CUP $-44.451, \mathrm{TIR}=5,70 \%$ y PRI $=7,67$ años.

Se obtuvieron correlaciones estadísticas que permiten relacionar cuantitativamente los indicadores VAN, TIR Y PRI con tres parámetros iniciales del proceso productivo: (i) capacidad de producción de cerveza por lote, (ii) costo de adquisición del sorgo rojo y (iii) precio de venta de la botella de cerveza.

\section{REFERENCIAS}

Aguiar, A. C. D. Osorio-Tobón, J. F., \& Silva, L. P. S. (2018). Economic analysis of oleoresin production from malagueta peppers (Capsicum frutescens) by supercritical fluid extraction. The Journal of Supercritical Fluids, 133, 86-93. https://doi. org/10.1016/j.supflu.2017.09.031

Alfonso, H. M. (2018). Estudio preliminar del proceso de obtención de cerveza a partir de sorgo rojo CIAP R-132 a escala de laboratorio (tesis de diploma, Universidad de Camagüey, Cuba). Repositorio Universidad de Camagüey.

Auli, N. A., Sakinah, M., Bakri, A. M. M. A., Kamarudin, H., \& Norazian, M. N. (2013). Simulation of xylitol production: A review. Australian Journal of Basic and Applied Sciences, 7(5), 366-372.

Baca, G. (2010). Evaluación de proyectos (6. ${ }^{\mathrm{a}}$ ed.). McGraw-Hill/Interamericana Editores, S.A. de C.V.

Carvajal, N. (2014). Perfeccionamiento del proceso de producción de cerveza a partir de malta de sorgo 
(tesis de diploma, Universidad Central Marta Abreu de Las Villas, Cuba). Repositorio Universidad Central Marta Abreu de las Villas.

Csighy, A., András, K., \& Vatai, G. (2017). Modelling the partial demineralization process of cow milk by SuperPro Designer. Hungarian Journal of Industry And Chemistry, 45(2), 9-12. https://doi. org/10.1515/hjic-2017-0013

Díaz, Y. (2014). Perfeccionamiento del proceso de malteado de sorgo para la producción de maltinas para enfermos celiacos (tesis de diploma, Universidad Central Marta Abreu de Las Villas, Cuba). Repositorio Universidad Central Marta Abreu de las Villas.

Ernst, S., Garro, O. A., Winkler, S., Venkataraman, G., Langer, R., Cooney, C. L., \& Sasisekharan, R. (1997). Process simulation for recombinant protein production: cost estimation and sensitivity analysis for heparinase I expressed in Escherichia coli. Biotechnology and Bioengineering, 53(6), 575-582. https://doi.org/10.1002/(SICl)10970290(19970320)53:6<575::AID-BIT5>3.0.CO;2-J

Gallardo, I., Boffill, Y., Ozuna, Y., Gómez, O., Pérez, M., \& Saucedo, O. (2013). Producción de bebidas usando sorgo malteado como materia prima para enfermos celíacos. Avances en Ciencias e Ingeniería, 4(1), 61-73.

Gallardo, I., Rodríguez, L., Boffill, Y., Alemán, L., \& Pérez, M. (2011). Producción de bioetanol empleando las enzimas generadas del sorgo malteado. Afinidad, 68(552), 144-149.

Govindarajan, D. K., Meganathan, Y., Udayakumar, G. P., \& Kothandan, R. (2019). Techno-economic analysis for the production of ethanol from Ipomoea batatas (sweet potato). American International Journal of Research in Science, Technology, Engineering \& Mathematics, February, 13-24.

Ibrahim, N. H., Ibrahim, W. H. W., Sakinah, A. M. M., Harun, N., \& Othman, M. R. (2020). Sensitivity analysis of xylose production process using aspen plus. IOP Conf. Series: Materials Science and Engineering, 736, 1-9. https://doi. org/10.1088/1757-899X/736/2/022110
Lam, K. F., Leung, C. C. J., Lei, H. M., \& Lin, C. S. K. (2014). Economic feasibility of a pilot-scale fermentative succinic acid production from bakery wastes. Food and Bioproducts Processing, 92, 282290. https://doi.org/10.1016/j.fbp.2013.09.001

Mani, S., Sundaram, J., \& Das, K. C. (2016). Process simulation and modeling: Anaerobic digestion of complex organic matter. Biomass and Bioenergy, 93, 158-167. https://doi.org/10.1016/j. biombioe.2016.07.018

Nieblas, C., Gallardo, I., Rodríguez, L., Carvajal, N., González, J. F., \& Pérez, M. (2016). Obtención de bebidas y otros productos alimenticios a partir de dos variedades de sorgo. Centro Azúcar, 43(3), 66-77.

Ortega, M. (2016). Producción de cerveza empleando integralmente el grano de sorgo (tesis de diploma, Universidad Central Marta Abreu de Las Villas, Cuba). Repositorio Universidad Central Marta Abreu de las Villas.

Pérez, E. J. (2016). Simulación del proceso de producción de cerveza a escala piloto (tesis de diploma, Universidad Central Marta Abreu de Las Villas, Cuba). Repositorio Universidad Central Marta Abreu de las Villas.

Perry, R. H., \& Green, D. W. (2008). Chemical Engineers' Handbook (8. ${ }^{\mathrm{a}}$ ed.). McGraw-Hill.

Peters, M. S., Timmerhaus, K. D., \& West, R. E. (2003). Plant design and economics for chemical engineers

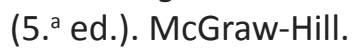

Pino, M. S. (2017). Tecnología para la producción de cerveza a partir de malta de sorgo para enfermos celíacos (tesis de diploma, Universidad Central Marta Abreu de Las Villas, Cuba). Repositorio Universidad Central Marta Abreu de las Villas.

Ramatoulaye, F., Mady, C., Fallou, S., Amadou, K., Cyril, D., \& Massamba, D. (2016). Production and use of sorghum: A literature review. Journal of Nutritional Health \& Food Science, 4(1), 1-4. https://doi.org/10.15226/jnhfs.2016.00157

Rao, S. R. (2005). Biomass to ethanol: process simulation, validation and sensitivity analysis 
of a gasifier and a bioreactor (tesis de maestría, Oklahoma State University). SHAREOK Repository.

Razi, N., Svendsen, H. F., \& Bolland, O. (2013). Cost and energy sensitivity analysis of absorber design in $\mathrm{CO}_{2}$ capture with MEA. International Journal of Greenhouse Gas Control, 10, 331-339. https://doi. org/10.1016/j.ijggc.2013.09.008

Rodríguez, L., Gallardo, I., Nieblas, C., Medina, J., \& Ortiz, W. (2015). Obtención de jarabes dextrinizados mediante hidrólisis enzimática del almidón de sorgo. Centro Azúcar, 42(4), 49-58.

Rodríguez, L., Gallardo, I., Nieblas, C., \& Ortiz, W. (2015). Evaluación de dos variedades de sorgo para la obtención de almidón. Centro Azúcar, 42(1), 88-95.

Sayar, N. A., Pinar, O., Kazan, D., \& Sayar, A. A. (2019). Bioethanol production from Turkish hazelnut husk process design and economic evaluation.
Waste Biomass Valor, 10, 909-923. https://doi. org/10.1007/s12649-017-0103-y

Sinnott, R., \& Towler, G. (2019). Chemical Engineering Design (6. ${ }^{a}$ ed., vol. 6). Elsevier ButterworthHeinemann.

Suárez, V. D., López, I., \& Álvarez, M. (2019). Estimación de la producción de biogás a partir de un modelo de simulación de procesos. Centro Azúcar, 46(1), 73-85.

Tang, Z.-C., Zhenzhou, L., Zhiwen, L., \& Ningcong, X. (2015). Uncertainty analysis and global sensitivity analysis of techno-economic assessments for biodiesel production. Bioresource Technology, 175, 502-508. https://doi.org/10.1016/j.biortech.2014.10.162

Towler, G., \& Sinnott, R. (2013). Chemical Engineering Design: Principles, Practice and Economics of Plant and Process Design (2. ${ }^{\mathrm{a}}$ ed.). Elsevier Ltd. 